\title{
On the zeros of Dirichlet $L$-functions
}

\author{
Sami Omar, Raouf Ouni and Kamel Mazhouda
}

\begin{abstract}
This paper [1], which was published online on 1 June 2011, has been retracted by agreement between the authors, the journal's Editor-in-Chief Derek Holt, the London Mathematical Society and Cambridge University Press. The retraction was agreed to prevent other authors from using incorrect mathematical results. (In this paper, we compute and verify the positivity of the $\mathrm{Li}$ coefficients for the Dirichlet $L$-functions using an arithmetic formula established in Omar and Mazhouda, J. Number Theory 125 (2007) no. 1, 50-58; J. Number Theory 130 (2010) no. 4, 1109-1114. Furthermore, we formulate a criterion for the partial Riemann hypothesis and we provide some numerical evidence for it using new formulas for the Li coefficients.)
\end{abstract}

\section{Introduction}

The Li criterion for the Riemann hypothesis (see [7]) is a necessary and sufficient condition that the sequence

$$
\left.\lambda_{n}=\sum_{\rho}\left[1-\left(1-\frac{1}{\rho}\right)^{n}\right]\right)
$$

is non-negative for all $n \in \mathbb{N}$ and where $\rho$ runs over the non-trivial zeros of $\zeta(s)$. This criterion holds also for the Dirichlet $L$-functions and for a large class of Dirichlet series, the socalled Selberg class as given in [11]. More recently, Omar and Bouanani [10] extended the $\mathrm{Li}$ criterion for function fields and established an explicit and asymptotic formula for the $\mathrm{Li}$ coefficients.

Numerical computation of the first 100 of the Li coefficients $\lambda_{n}$ which appear in this criterion was made by Maślanka in [9] and later by Coffey [3], who computed and verified the positivity of about 3300 of the Li coefficients $\lambda_{n}$. The main empirical observation made by Maślanka is that these coefficients can be separated into two parts, where one of them grows smoothly while the other is very small and oscillatory. This apparent smallness is quite unexpected. If it persisted until infinity then the Riemann hypothesis would be true. As we said above, this criterion was extended to a large class of Dirichlet series [11] and no calculation or verification of the positivity to date in the literature was made for other $L$-functions.

In this paper, we compute and verify the positivity of the Li coefficients for the Dirichlet $L$-functions using an arithmetic formula established in $[\mathbf{1 1}, \mathbf{1 2}]$. Furthermore, we formulate a criterion for the partial Riemann hypothesis. Additional results are presented, including new formulas for the Li coefficients. Based on the numerical computations made below, we conjecture that these coefficients are increasing in $n$. Should this conjecture hold, the validity of the Riemann hypothesis would follow.

Next, we review the Li criterion for the case of the Dirichlet $L$-functions. Let $\chi$ be a primitive Dirichlet character of conductor $q$. The Dirichlet $L$-function attached to this character is defined by

$$
L(s, \chi)=\sum_{n=1}^{\infty} \frac{\chi(n)}{n^{s}} \quad(\operatorname{Re}(s)>1) .
$$

Received 19 June 2010; revised 5 November 2010.

2000 Mathematics Subject Classification 11M06 (primary), 11M26, $11 \mathrm{M} 36$ (secondary). 
For the trivial character $\chi=1, L(s, \chi)$ is the Riemann zeta function. It is well known [4] that if $\chi \neq 1$ then $L(s, \chi)$ can be extended to an entire function in the whole complex plane and satisfies the functional equation

$$
\xi(s, \chi)=\omega_{\chi} \xi(1-s, \bar{\chi})
$$

where

$$
\begin{gathered}
\xi(s, \chi)=\left(\frac{q}{\pi}\right)^{(s+a) / 2} \Gamma\left(\frac{s+a}{2}\right) L(s, \chi), \\
a=\left\{\begin{array}{ll}
0 & \text { if } \chi(-1)=1, \\
1 & \text { if } \chi(-1)=-1,
\end{array} \quad \text { and } \quad \omega_{\chi}=\frac{\tau(\chi)}{\sqrt{q} i^{a}},\right.
\end{gathered}
$$

where $\tau(\chi)$ is the Gauss sum

$$
\tau(\chi)=\sum_{m=1}^{q} \chi(m) e^{2 \pi i m / q} .
$$

The function $\xi(s, \chi)$ is an entire function of order one. The function $\xi(s, \chi)$ has a product representation

$$
\xi(s, \chi)=\xi(0, \chi) \prod_{\rho}\left(1-\frac{s}{\rho}\right),
$$

where the product is over all the zeros of $\xi(s, \chi)$ in the order given by $|\operatorname{Im}(\rho)|<T$ for $T \rightarrow \infty$. If $N_{\chi}(T)$ counts the number of zeros of $L(s, \chi)$ in the rectangle $0 \leqslant \operatorname{Re}(s) \leqslant 1,0<\operatorname{Im}(s) \leqslant T$ (according to multiplicities), one can show by standard contour integration the formula

$$
N_{\chi}(T)=\frac{1}{2 \pi} T \log T+c_{1} T+O(\log T)
$$

where

We put

$$
c_{1}=\frac{1}{2 \pi}(\log q-(\log (2 \pi)+1)) \text {. }
$$

$$
\lambda_{\chi}(n)=\sum_{\rho}\left[1-\left(1-\frac{1}{\rho}\right)^{n}\right],
$$

where the sum over $\rho$ is $\sum_{\rho}=\lim _{T \mapsto \infty} \sum_{|\operatorname{Im}(\rho)| \leqslant T}$.

Li's criterion says that $\lambda_{\chi}(n)>0$ for all $n=1,2, \ldots$ if and only if all of the zeros of $\xi(s, \chi)$ are located on the critical line $\operatorname{Re}(s)=1 / 2$.

The paper is organized as follows. In Section 2, we recall the arithmetic formula for the $\mathrm{Li}$ coefficients for the Dirichlet $L$-functions and we give an estimate for the error term of $\lambda_{\chi}(n)$. In Section 3, we show that $\lambda_{\chi}(n) \geqslant 0$ if every non-trivial zero of $L(s, \chi)$ with $|\operatorname{Im}(\rho)|<\sqrt{n}$ satisfies $\operatorname{Re}(\rho)=1 / 2$ (that is the partial Riemann hypothesis) and we give an estimate for the difference $\left|\lambda_{\chi}(n)-\lambda_{\chi}(n, T)\right|$, where $\lambda_{\chi}(n, T)$ are the partial Li coefficients. In Section 4, we prove new formulas (integral and summation formulas) for the Li coefficients $\lambda_{\chi}(n)$. Finally, in Section 5, we report numerical computations of the Li coefficients using different formulas established in the previous sections unconditionally or under the Riemann hypothesis.

\section{Li's coefficients}

Applying [11, Theorem 2.2] for the case of the Dirichlet $L$-functions, we get the following arithmetic formula. 
Theorem 1. Let $\chi$ be a primitive Dirichlet character of conductor $q>1$. We have

$$
\begin{aligned}
\lambda_{\chi}(n)= & -\sum_{j=1}^{n}\left(\begin{array}{c}
n \\
j
\end{array}\right) \frac{(-1)^{j-1}}{(j-1) !} \sum_{k=1}^{+\infty} \frac{\Lambda(k)}{k} \chi(k)(\log k)^{j-1} \\
& +\frac{n}{2}\left(\log \frac{q}{\pi}-\gamma\right)+\tau_{\chi}(n),
\end{aligned}
$$

where

$$
\tau_{\chi}(n)= \begin{cases}\sum_{j=2}^{n}\left(\begin{array}{l}
n \\
j
\end{array}\right)(-1)^{j}\left(1-\frac{1}{2^{j}}\right) \zeta(j)-\frac{n}{2} \sum_{l=1}^{+\infty} \frac{1}{l(2 l-1)} & \text { if } \chi(-1)=1, \\
\sum_{j=2}^{n}\left(\begin{array}{l}
n \\
j
\end{array}\right)(-1)^{j} 2^{-j} \zeta(j) & \text { if } \chi(-1)=-1 .\end{cases}
$$

Theorem 1 was also proved by Coffey in [3] and Li in [8]. The arithmetic formula above can be written as

$$
\begin{aligned}
\lambda_{\chi}(n)= & {\left[\log \frac{q}{\pi}+\psi\left(\frac{a+1}{2}\right)\right] \frac{n}{2}+\sum_{j=2}^{n}\left(\begin{array}{l}
n \\
j
\end{array}\right) \frac{1}{(j-1) !} 2^{-j} \psi^{(j-1)}\left(\frac{a+1}{2}\right) } \\
& -\sum_{j=1}^{n}\left(\begin{array}{c}
n \\
j
\end{array}\right) \frac{(-1)^{j-1}}{(j-1) !} \sum_{k=1}^{+\infty} \frac{\Lambda(k) \chi(k)}{k}(\log k)^{j-1},
\end{aligned}
$$

where $a=0$ if $\chi(-1)=1$ and 1 if $\chi(-1)=-1$, with $\psi\left(\frac{1}{2}\right)=-\gamma-2 \log 2, \psi(1)=-\gamma(\gamma$ is the Euler constant), $\psi^{(j-1)}(1)=(-1)^{j}(j-1) ! \zeta(j)$ and $\psi^{(j-1)}\left(\frac{1}{2}\right)=(-1)^{j+1} j !\left(2^{j+1}-1\right) \zeta(j+1)$. Here, $\psi=\Gamma^{\prime} / \Gamma$ denotes the digamma function.

An asymptotic formula for the number $\lambda_{\chi}(n)$ was proved in [13, Theorem 3.1] using the arithmetic formula. Furthermore, it is equivalent to the Riemann hypothesis.

Theorem 2. We have

$$
\mathrm{RH} \Leftrightarrow \lambda_{\chi}(n)=\frac{1}{2} n \log n+c_{\chi} n+O(\sqrt{n} \log n),
$$

where

$$
c_{\chi}=\frac{1}{2}(\gamma-1)+\frac{1}{2} \log (q / \pi)
$$

and $\gamma$ is the Euler constant.

Here, we estimate the error term for $\lambda_{\chi}(n)$ using the arithmetic formula (2). We write (2) in the form

$$
\lambda_{\chi}(n)=\tilde{\lambda}_{\chi}(n, M)+E_{M}
$$

where

$$
\tilde{\lambda}_{\chi}(n, M)=\frac{n}{2}\left(\log \frac{q}{\pi}-\gamma\right)+\tau_{\chi}(n)-\sum_{j=1}^{n}\left(\begin{array}{l}
n \\
j
\end{array}\right) \frac{(-1)^{j-1}}{(j-1) !} \sum_{k \leqslant M} \frac{\Lambda(k)}{k} \chi(k)(\log k)^{j-1}
$$

and

$$
E_{M}=-\sum_{j=1}^{n}\left(\begin{array}{l}
n \\
j
\end{array}\right) \frac{(-1)^{j-1}}{(j-1) !} \sum_{k>M} \frac{\Lambda(k)}{k} \chi(k)(\log k)^{j-1}=-\sum_{k>M} \frac{\Lambda(k)}{k} \chi(k) L_{n-1}^{1}(\log k),
$$

where $L_{n-1}^{1}$ is an associated Laguerre polynomial of degree $n-1$. 
Next, for our computation in Section 5, we need to find $M$ such that $\left|E_{M}\right| \leqslant 10^{-\nu}$. An estimate for the Laguerre polynomials is due to Koepf and Schmersau [6, Theorem 2]. Actually, they have shown that

$$
\left|L_{n}^{\alpha}(x)\right|<e^{x / 2}[(n+\alpha) / x]^{\alpha / 2}
$$

for $x \in[0,4(n+\alpha)]$ when $n+\alpha>0$ and $\alpha$ is an integer. Then we obtain

$$
\left|E_{M}\right| \leqslant \sqrt{\frac{n}{\log M}} \sum_{m>M}^{+\infty} \frac{\Lambda(m)}{m}|\chi(m)| \leqslant \sqrt{\frac{n}{\log M}} \sum_{p^{j}>M}^{+\infty} \frac{\log p}{p^{j}}\left|\chi\left(p^{j}\right)\right| .
$$

We have $\left|\chi\left(p^{j}\right)\right|<1$. Therefore,

$$
\left|E_{M}\right| \leqslant \sqrt{\frac{n}{\log M}} \sum_{p^{j}>M}^{+\infty} \frac{\log p}{p^{j}} \leqslant \begin{cases}\sqrt{\frac{n}{\log M} \frac{\log M}{M} \leqslant \sqrt{n} \frac{\log M}{M}} \quad \text { if } M+1 \text { is prime } \\ \sqrt{\frac{n}{\log M} \frac{1}{M} \leqslant \frac{\sqrt{n}}{M}} \quad \text { otherwise. }\end{cases}
$$

Let $M$ be such that $\left|E_{M}\right| \leqslant 10^{-\nu}$. Then

$$
\begin{cases}\sqrt{n} \frac{\log M}{M} \leqslant 10^{-\nu} & \text { if } M+1 \text { is prime } \\ \frac{\sqrt{n}}{M} \leqslant 10^{-\nu} & \text { otherwise. }\end{cases}
$$

Using the theory of the Lambert $W$ function, we choose

$$
M= \begin{cases}-\sqrt{n} W_{-1}\left(-\frac{10^{-\nu}}{\sqrt{n}}\right) & \text { if } M+1 \text { is prime } \\ \sqrt{n} 10^{\nu} & \text { otherwise }\end{cases}
$$

where $W_{-1}$ denotes the branch satisfying $W(x) \leqslant-1$ and $W(x)$ is the Lambert $W$ function, which is defined to be the multivalued inverse of the function $w \longmapsto w e^{w}$.

\section{Partial Li criterion}

In the following proposition, we propose a partial Li criterion which relates the partial Riemann hypothesis to the positivity of the Li coefficients up to a certain order.

Proposition 1. If every non-trivial zero $\rho$ of $L(s, \chi)$ with $|\operatorname{Im}(\rho)|<T^{2}$ satisfies $\operatorname{Re}(\rho)=$ $1 / 2$, then $\lambda_{\chi}(n) \geqslant 0$ for all $n \leqslant T$.

Proof. We have

$$
\lambda_{\chi}(n)=\sum_{\rho}\left[1-\left(1-\frac{1}{\rho}\right)^{n}\right]
$$

Note that there are $O(\log u)$ zeros in $[u, u+1]$. Then

$$
\begin{aligned}
\lambda_{\chi}(n)= & \sum_{\rho}\left[1-\operatorname{Re}\left(1-\frac{1}{\rho}\right)^{n}\right] \\
= & \sum_{\rho ;|\operatorname{Im}(\rho)|<T^{2}}\left[1-\operatorname{Re}\left(1-\frac{1}{\rho}\right)^{n}\right]+\sum_{\rho ; T^{2}<|\operatorname{Im}(\rho)|<T^{2}(\log T)^{2}}\left[1-\operatorname{Re}\left(1-\frac{1}{\rho}\right)^{n}\right] \\
& +\sum_{\rho ;|\operatorname{Im}(\rho)|>T^{2}(\log T)^{2}}\left[1-\operatorname{Re}\left(1-\frac{1}{\rho}\right)^{n}\right] .
\end{aligned}
$$


Let $\rho=\beta+i \gamma$; then we obtain

$$
1-\left(1-\frac{1}{\rho}\right)^{n}=1-\left(\frac{1+(\beta-1) / i \gamma}{1+\beta / i \gamma}\right)^{n}, \quad \frac{1+(\beta-1) / i \gamma}{1+\beta / i \gamma}=\left(1-\frac{\beta}{\gamma^{2}}\right)+\frac{i}{\gamma}+O\left(\frac{1}{\gamma^{3}}\right)
$$

and

$$
\operatorname{Re}\left(\frac{1+(\beta-1) / i \gamma}{1+\beta / i \gamma}\right)^{n}=\left(1-\frac{\beta}{\gamma^{2}}\right)^{n}+O\left(\frac{1}{\gamma^{4}}\right)
$$

Therefore,

$$
\begin{aligned}
\sum_{|\gamma|>T^{2} \log T}\left[1-\operatorname{Re}\left(1-\frac{1}{\rho}\right)^{n}\right] & =\sum_{|\gamma|>T^{2} \log T}\left[1-\left(1-\frac{\beta}{\gamma^{2}}\right)^{n}+O\left(\frac{1}{\gamma^{4}}\right)\right] \\
& =\sum_{\substack{|\gamma|>T^{2} \log T\\
}} \frac{n \beta}{\gamma^{2}}+O\left(\frac{1}{\gamma^{4}}\right) \\
& =O(1) .
\end{aligned}
$$

And,

$$
\begin{aligned}
\sum_{T^{2}<|\gamma|<T^{2}(\log T)^{2}}\left[1-\operatorname{Re}\left(1-\frac{1}{\rho}\right)^{n}\right] & =\sum_{T^{2}<|\gamma|<T^{2}(\log T)^{2}}\left[1-\left(1-\frac{\beta}{\gamma^{2}}\right)^{n}+O\left(\frac{1}{\gamma^{4}}\right)\right] \\
& =\sum_{T^{2}<|\gamma|<T^{2}(\log T)^{2}} \frac{n \beta}{\gamma^{2}}+O\left(\frac{1}{\gamma^{4}}\right) \\
& =O\left((\log T)^{3}\right) .
\end{aligned}
$$

Indeed, since $n \leqslant T$, we obtain

$$
\begin{aligned}
\sum_{T^{2}<|\gamma|<T^{2}(\log T)^{2} \gamma^{2}} \frac{n \beta}{\gamma^{2}} & \ll(\log T)\left[\sum_{j=T^{2}}^{T^{2}(\log T)^{2}} \frac{n}{j}\right] \\
& \ll T \log T\left[\sum_{j=T^{2}}^{T^{2}(\log T)^{2}} \frac{1}{j}\right] \\
& \ll T \log T\left[\frac{(\log T)^{2}}{T}\right] \\
& \ll(\log T)^{3} .
\end{aligned}
$$

Finally, it suffices to prove that, under the Riemann hypothesis, there exists a positive constant $c_{0}$ such that

$$
\sum_{|\operatorname{Im}(\rho)|<T^{2}}\left[1-\operatorname{Re}\left(1-\frac{1}{\rho}\right)^{n}\right] \geqslant c_{0} T \log T .
$$

Thus,

$$
\begin{aligned}
\sum_{|\gamma|<T^{2}}\left[1-\operatorname{Re}\left(1-\frac{1}{\rho}\right)^{n}\right] & \geqslant \sum_{|\gamma|<T^{2}} \frac{n^{2}}{2 \gamma^{2}} \\
& \geqslant \frac{T^{2}}{2} \sum_{|\gamma|<T} \frac{1}{\gamma^{2}} \\
& \geqslant \frac{1}{2} N_{\chi}(T) .
\end{aligned}
$$


Recall that

$$
N_{\chi}(T)=\frac{1}{2 \pi} T \log T+c_{1} T+O(\log T)
$$

Then (10) is proved.

REMARK.

- Recall that the $10^{13}$ first zeros of the Riemann zeta function lie on the line $\operatorname{Re}(s)=1 / 2$ (see [5]). Then, from Proposition 1, the first $10^{6} \mathrm{Li}$ coefficients $\lambda_{\zeta}(n)$ are non-negative.

- In Section 5, we will use the first $10^{4}$ critical zeros of the Dirichlet $L$-functions to compute the first Li coefficients $\lambda_{\chi}(n)$. Then, from Proposition 1 above, we affirm that the first 100 Li coefficients are non-negative.

Conversely. From the work of Brown [2], the first observation is that the first 'non-trivial' inequality $\lambda_{\chi}(2) \geqslant 0$ is sufficient to establish the non-existence of a Siegel zero for $\xi(s, \chi)$ (see [2, Corollary 1]).

Let $r>1$ be a real number. By the invariance of the zeros $\rho$ of $\xi(s, \chi)$ under the map $\rho \longmapsto 1-\bar{\rho}$,

$$
\forall \rho, \quad\left|\frac{\rho}{\rho-1}\right| \leqslant r \Leftrightarrow \forall \rho, \rho \in D_{r}
$$

where $D_{r}$ is the closed region bounded by the lines $\{z \in \mathbb{C}: \operatorname{Re}(z)=0,1\}$ and the arcs of two circles. The second observation (see $[\mathbf{2}$, Theorem 3]) is that, for large $N \in \mathbb{N}$, the inequalities $\lambda_{\chi}(1) \geqslant 0, \ldots, \lambda_{\chi}(n) \geqslant 0$ imply the existence of a certain zero-free region for $\xi(s, \chi)$, that is, there exist constants $N, \mu, \nu$ depending only on $q$ such that if $\lambda_{\chi}(1) \geqslant 0, \ldots, \lambda_{\chi}(n) \geqslant 0$ hold, and $n \geqslant N$, then the zeros of $\xi(s, \chi)$ belong to $D_{r}$, where $r=\sqrt{1+T^{-2}}$ and $T=$ $\left(n / \mu \log ^{2}(\nu n)\right)^{1 / 3}$.

Let us define the partial Li coefficients by

$$
\lambda_{\chi}(n, T)=\sum_{\rho ;|\operatorname{Im} \rho| \leqslant T} 1-\left(1-\frac{1}{\rho}\right)^{n}
$$

with a parameter $T$. An estimate for the error term $\left|\lambda_{\chi}(n)-\lambda_{\chi}(n, T)\right|$ is stated in the following proposition.

Proposition 2. We have

$$
\left|\lambda_{\chi}(n)-\lambda_{\chi}(n, T)\right| \leqslant \frac{3 n^{2}}{2 T^{2}}\left[\frac{1}{2 \pi} T \log T+\left(\frac{1}{\pi}+\log \left(\frac{q}{2 \pi e}\right)\right) T+\frac{1}{2}\right] .
$$

Proof. Note that $\rho=\beta+i \gamma$, where $\beta, \gamma \in \mathbb{R}$ and $0 \leqslant \beta \leqslant 1$. We have

$$
\lambda_{\chi}(n)-\lambda_{\chi}(n, T)=\frac{1}{2} \operatorname{Re}\left[\sum_{|\gamma|>T}\left(2-\left(\frac{\rho-1}{\rho}\right)^{n}-\left(\frac{\rho}{\rho-1}\right)^{n}\right)\right] .
$$

Using a binomial expansion of the inner term in the sum in the right-hand side, we obtain

$$
\begin{aligned}
\operatorname{Re}\left[2-\left(\frac{\rho-1}{\rho}\right)^{n}-\left(\frac{\rho}{\rho-1}\right)^{n}\right]= & \operatorname{Re}\left[n\left(\frac{1}{\rho}+\frac{1}{1-\rho}\right)-\frac{n(n-1)}{2}\left(\frac{1}{\rho^{2}}+\frac{1}{(1-\rho)^{2}}\right)\right] \\
& +\operatorname{Re}\left[\sum_{k=3}^{n}\left(\begin{array}{l}
n \\
k
\end{array}\right)(-1)^{k-1}\left(\rho^{-k}+(1-\rho)^{-k}\right)\right]
\end{aligned}
$$

We have

$$
\frac{1}{1+\gamma^{2}} \leqslant \operatorname{Re}\left(\frac{1}{\rho}+\frac{1}{1-\rho}\right) \leqslant \frac{1}{\gamma^{2}}
$$


and

$$
\frac{1}{1+\gamma^{2}}-\frac{2}{\gamma^{4}} \leqslant \operatorname{Re}\left(\frac{1}{\rho^{2}}+\frac{1}{(1-\rho)^{2}}\right) \leqslant \frac{2}{\gamma^{2}} .
$$

Suppose now that $|\gamma| \geqslant T \geqslant n$; then $(n-3) /|\gamma| \ldots(n-k) /|\gamma| \leqslant 1$ for all $n \geqslant k \geqslant 3$. Then

$$
\sum_{k=3}^{n}\left(\begin{array}{l}
n \\
k
\end{array}\right) \frac{1}{|\gamma|^{k}} \leqslant 2 \frac{n^{3}}{|\gamma|^{3}} \sum_{k=3}^{\infty} \frac{1}{k !}=(2 e-5) \frac{n^{3}}{|\gamma|^{3}} \leqslant \frac{n^{3}}{2|\gamma|^{3}} .
$$

Therefore,

$$
\begin{aligned}
\operatorname{Re}\left[2-\left(\frac{\rho-1}{\rho}\right)^{n}-\left(\frac{\rho}{\rho-1}\right)^{n}\right] & \leqslant \frac{n}{\gamma^{2}}+\frac{n^{2}-n}{\gamma^{2}}+\sum_{k=3}^{n}\left(\begin{array}{l}
n \\
k
\end{array}\right) \frac{2}{|\gamma|^{k}} \\
& \leqslant \frac{n}{\gamma^{2}}+\frac{n^{3}}{2|\gamma|^{3}} \\
& \leqslant \frac{3 n^{2}}{2|\gamma|^{2}}
\end{aligned}
$$

Then

We have

$$
\left|\lambda_{\chi}(n)-\lambda_{\chi}(n, T)\right| \leqslant \frac{3}{4} n^{2} \sum_{|\gamma|>T} \frac{1}{\gamma^{2}}
$$

$$
\frac{1}{2} \sum_{|\gamma|>T} \frac{1}{\gamma^{2}} \leqslant \int_{T}^{\infty}-\frac{d}{d t}\left[t^{-2}\right]_{t=x}\left(N_{\chi}(x)-N_{\chi}(T)\right) d x=\int_{T}^{\infty} x^{-2} d N_{\chi}(x) .
$$

Furthermore,

$$
\begin{aligned}
\int_{T}^{\infty} x^{-2} d N_{\chi}(x) & =\int_{T}^{\infty} x^{-2}\left[\frac{1}{2 \pi} \log x+\frac{1}{2 \pi}+\log \left(\frac{q}{2 \pi e}\right)+\frac{1}{x}\right] d x \\
& =T^{-2}\left[\frac{1}{2 \pi} \log T+\frac{1}{2 \pi} T \log T+\frac{1}{2 \pi} T+\log \left(\frac{q}{2 \pi e}\right) T+\frac{1}{2}\right] .
\end{aligned}
$$

Finally, we get

$$
\left|\lambda_{\chi}(n)-\lambda_{\chi}(n, T)\right| \leqslant \frac{3}{2} \frac{n^{2}}{T^{2}}\left[\frac{1}{2 \pi} T \log T+\left(\frac{1}{\pi}+\log \left(\frac{q}{2 \pi e}\right)\right) T+\frac{1}{2}\right]
$$

and Proposition 2 follows.

For our computations at the end of this paper, we need to find $T_{0}$ such that $\mid \lambda_{\chi}(n)-$ $\lambda_{\chi}(n, T) \mid \leqslant 10^{-k}$. To do so, it suffices to find $T_{0}$ such that

$$
\frac{3 n^{2}}{4 \pi} \frac{\log T}{T} \leqslant \frac{10^{-k}}{3} \Leftrightarrow \frac{\log T}{T} \leqslant \frac{4 \pi 10^{-k}}{9 n^{2}}
$$

Using the theory of the Lambert $W$ function, we get

$$
T_{0}=-\frac{9 n^{2}}{4 \pi} W_{-1}\left(-\frac{4 \pi}{9 n^{2}} 10^{-k}\right)
$$

where $W_{-1}$ denotes the branch satisfying $W(x) \leqslant-1$ and $W(x)$ is the Lambert $W$ function, which is defined to be the multivalued inverse of the function $w \longmapsto w e^{w}$.

\section{New formulas for the Li coefficients}

In this section, we give new formulas for the Li coefficients under the Riemann hypothesis (integral and summation formulas) which will be used to compute and verify the positivity of the Li coefficients $\lambda_{\chi}(n)$ under the Riemann hypothesis. 
From (1), we have

$$
\log \xi\left(\frac{z}{z-1}, \chi\right)=\log \xi\left(\frac{1}{1-z}, \chi\right)=\log \xi(0, \chi)+\sum_{n=1}^{\infty} \lambda_{\chi}(n) \frac{z^{n}}{n} .
$$

The number $\lambda_{\chi}(n)$ does not depend on the choice of the logarithm. Rewrite (16) at the point $z=-1$. Note that the region of convergence for this is an open disk of radius 2 centered at $z=-1$ and it encloses in particular the entirety of the closed unit disk, except for the point $z=1$ that is a pole of $\xi(1 /(1-z), \chi)$.

Assume that the Riemann hypothesis holds. Then we have

$$
\begin{aligned}
\log \xi\left(\frac{1}{1-z}, \chi\right) & =\log \xi(0, \chi)+\sum_{n=1}^{\infty} \lambda_{\chi}(n) \frac{z^{n}}{n} \\
& =C_{\chi}(0)+\sum_{n=1}^{\infty} C_{\chi}(n)(z+1)^{n}
\end{aligned}
$$

Expanding $(z+1)^{n}$, we obtain

$$
\lambda_{\chi}(n)=n \sum_{j=1}^{\infty}\left(\begin{array}{l}
j \\
n
\end{array}\right) C_{\chi}(j)
$$

We have

$$
N_{\chi}(T)=\frac{1}{\pi} \operatorname{Im}\left(\log \xi_{\chi}\left(\frac{1}{2}+i T\right)\right)=\sum_{n=1}^{\infty} \frac{C_{\chi}(n)}{\pi}\left(\operatorname{Im}\left(\frac{2 \gamma+i}{2 \gamma-i}+1\right)\right)^{n},
$$

where we have used the substitution $\frac{1}{2}+i \gamma=1 /(1-z)$ or $z=(2 \gamma+i) /(2 \gamma-i)$. Then, since

$$
\begin{gathered}
\left(\operatorname{Im}\left(\frac{2 \gamma+i}{2 \gamma-i}+1\right)\right)^{n}=\frac{(4 \gamma)^{n}}{\left(4 \gamma^{2}+1\right)^{n / 2}} \sin \left(n \tan ^{-1} \frac{1}{2 \gamma}\right)=2^{n} \cos ^{n} \theta \sin (n \theta) \\
\cos \theta:=\frac{2 \gamma}{\sqrt{4 \gamma^{2}+1}},
\end{gathered}
$$

we get

$$
\pi N_{\chi}(\gamma)=\sum_{n=1}^{\infty} C_{\chi}(n) 2^{n} \cos ^{n} \theta \sin (n \theta)
$$

Using the identity

we deduce that

$$
\int_{0}^{\pi / 2} \cos ^{n} \theta \sin (n \theta) \sin (2 m \theta) d \theta=\frac{\pi}{2^{n+2}}\left(\begin{array}{c}
n \\
m
\end{array}\right), \quad m, n \in \mathbb{N}
$$

$$
\int_{0}^{\pi / 2} \pi N_{\chi}(\gamma) \sin (2 m \theta) d \theta=\sum_{n=1}^{\infty} C_{\chi}(n) \frac{\pi}{4}\left(\begin{array}{c}
n \\
m
\end{array}\right) .
$$

Hence,

$$
\sum_{n=1}^{\infty} C_{\chi}(n)\left(\begin{array}{c}
n \\
m
\end{array}\right)=4 \int_{0}^{\pi / 2} N_{\chi}(\gamma) U_{m-1}(\cos (2 \theta)) \sin (2 \theta) d \theta,
$$

where $U_{m-1}$ are the Chebyschev polynomials of the second kind. Using

$$
\begin{aligned}
U_{m-1}(\cos \theta):= & \frac{\sin (m \theta)}{\sin (\theta)}, \quad \cos (2 \theta)=\cos ^{2} \theta-\sin ^{2} \theta=\frac{4 \gamma^{2}-1}{4 \gamma^{2}+1}, \\
& \sin (2 \theta) d \theta=-2 \cos \theta d(\cos \theta)
\end{aligned}
$$


and that as $\gamma$ proceeds from 0 to $\infty, \theta$ subtends an angle from $\pi / 2$ to 0 , we obtain

$$
\sum_{n=1}^{\infty} C_{\chi}(n)\left(\begin{array}{c}
n \\
m
\end{array}\right)=8 \int_{0}^{\infty} N_{\chi}(\gamma) U_{m-1}\left(\frac{4 \gamma^{2}-1}{4 \gamma^{2}+1}\right) \times \frac{2 \gamma}{\sqrt{4 \gamma^{2}+1}} \times \frac{2}{\left(4 \gamma^{2}+1\right)^{3 / 2}} d \gamma .
$$

Therefore, from (18) we get for all $n \in \mathbb{N}$ the following proposition.

Proposition 3. Under the Riemann hypothesis, we have

$$
\lambda_{\chi}(n)=32 n \int_{0}^{\infty} \frac{\gamma}{\left(4 \gamma^{2}+1\right)^{2}} N_{\chi}(\gamma) U_{n-1}\left(\frac{4 \gamma^{2}-1}{4 \gamma^{2}+1}\right) d \gamma
$$

Next, we give another formula for the Li coefficients. Recall that the function $N_{\chi}(T)$ is a real step function, increasing by unity each time a new critical zero is counted:

$$
N_{\chi}(T)=\sum_{\rho, \operatorname{Im}(\rho)>0} \phi(T-\operatorname{Im}(\rho))=\sum_{k=1}^{\infty} \alpha_{k} \phi\left(T-\gamma_{k}\right),
$$

where $\rho_{j}=\beta_{k}+i \gamma_{k}, \gamma_{k}>0$ and $\phi(x-a)=1$ if $x \geqslant a$ and 0 if $x<a$. The zeros are ordered so that $\gamma_{k+1}>\gamma_{k}$ and the $\alpha_{k}$ count the number of zeros with imaginary part $\gamma_{k}$ including the multiplicities. Simplification of the integral formula (20) is stated in the following proposition.

Proposition 4. Under the Riemann hypothesis, we have

$$
\lambda_{\chi}(n)=2 \sum_{k=1}^{\infty} \alpha_{k}\left(1-T_{n}\left(\frac{4 \gamma_{k}^{2}-1}{4 \gamma_{k}^{2}+1}\right)\right), \quad n \in \mathbb{N} .
$$

Proof. By (21), the formula (20) can be written as follows:

$$
\begin{aligned}
\lambda_{\chi}(n) & =32 n \sum_{k=1}^{\infty} \alpha_{k} \int_{0}^{\infty} \phi\left(\gamma-\gamma_{k}\right) \frac{\gamma}{\left(4 \gamma^{2}+1\right)^{2}} U_{n-1}\left(\frac{4 \gamma^{2}-1}{4 \gamma^{2}+1}\right) d \gamma \\
& =2 n \sum_{k=1}^{\infty} \alpha_{k} \int_{\gamma_{k}}^{\infty} \frac{16 \gamma}{\left(4 \gamma^{2}+1\right)^{2}} U_{n-1}\left(\frac{4 \gamma^{2}-1}{4 \gamma^{2}+1}\right) d \gamma \\
& =2 n \sum_{k=1}^{\infty} \alpha_{k}\left[\frac{1}{n} T_{n}(y)\right]_{\left(4 \gamma_{k}^{2}-1\right) /\left(4 \gamma_{k}^{2}+1\right)}^{1} \\
& =2 \sum_{k=1}^{\infty} \alpha_{k}\left(1-T_{n}\left(\frac{4 \gamma_{k}^{2}-1}{4 \gamma_{k}^{2}+1}\right)\right),
\end{aligned}
$$

using the following relation between the Chebyshev polynomials of the second kind and the first kind:

$$
\int U_{n}(x) d x=\frac{1}{n+1} T_{n+1}(x)
$$

This is a remarkable summation expression for the Li coefficients. We numerically evaluate some of the first terms by the right-hand-side expression and find them to be indeed close to the required values of the Li coefficients. This is reassuring, and the results are presented in the tables below.

Under the Riemann hypothesis, from the above arguments used in the proof of Propositions 3 and 4 , one can derive the following formula:

$$
\lambda_{\chi}(n, T)=2 \sum_{k=1}^{N} \alpha_{k}\left(1-T_{n}\left(\frac{4 \gamma_{k}^{2}-1}{4 \gamma_{k}^{2}+1}\right)\right),
$$


where $N=\left[N_{\chi}(T)\right]$ with $[x]=x-\{x\}$ and $\{x\}$ denotes the fractional part of $x$ (the last formula will be denoted $\left.\lambda_{\chi}(n, N)\right)$. Therefore, the latter formula allows one to estimate the error term $\left|\lambda_{\chi}(n)-\lambda_{\chi}(n, N)\right|$ in Proposition 4 by evaluating directly the partial Li coefficients as in Proposition 2.

\section{Numerical computations}

In this section, we compute and verify the positivity of the values of $\lambda_{\chi}(n)$ unconditionally or under the Riemann hypothesis. We first compute unconditionally (without assuming the Riemann hypothesis) $\tilde{\lambda}_{\chi}(n, M)$ by using (3) and computing prime numbers up to $M$ (see Section 2). We also compute under the Riemann hypothesis

$$
\lambda_{\chi}(n, N)=2 \sum_{k=1}^{N} \alpha_{k}\left(1-T_{n}\left(\frac{4 \gamma_{k}^{2}-1}{4 \gamma_{k}^{2}+1}\right)\right) \quad \text { with } N=10^{4}
$$

Furthermore, we carried out the calculations for several examples of characters. Some illustrative examples are cited below. We restricted the tables below for $n \leqslant 40$. However, one can find the other values of $n>40$ represented in Figures 1-4

REMARK. In fact, by the summation formula (22), we could compute more coefficients $\lambda_{\chi}(n)$ in a less time consuming way than by the arithmetic formula (3), where computation of the first 50 coefficients lasted more than a week.

Based on the tables below, we conjecture the following result.

Conjecture. The coefficients $\lambda_{\chi}(n)$ are positive and increasing in $n$.

This conjecture was partially numerically verified for the case of the Riemann zeta function (see [3, Appendix D] and [9]) and by the authors in a work in progress for the Hecke $L$-functions [14].

Acknowledgement. The authors would like to thank Jeffery Lagarias for helpful comments and especially in the proof of Proposition 1. 


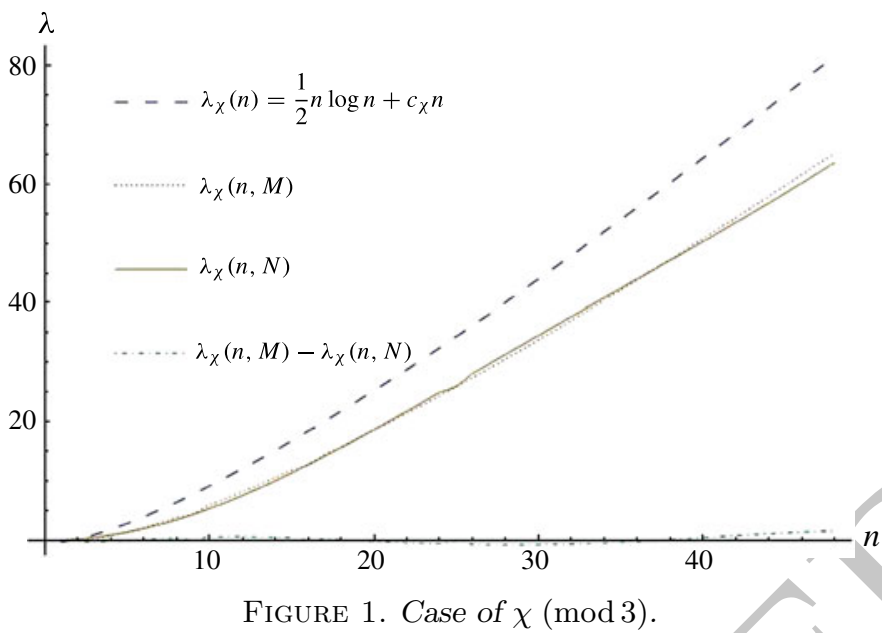

\begin{tabular}{rrrrrl}
\hline \multicolumn{5}{c}{$\chi(\bmod 3)$} & \\
\hline$n$ & $\tilde{\lambda}_{\chi}(n, M)$ & $\lambda_{\chi}(n, N)$ & $n$ & $\tilde{\lambda}_{\chi}(n, M)$ & $\lambda_{\chi}(n, N)$ \\
\hline 1 & 0.05316 & 0.056442 & 19 & 17.18050 & 17.16170 \\
2 & 0.22763 & 0.22542 & 20 & 18.58480 & 18.69100 \\
3 & 0.14844 & 0.50592 & 21 & 20.01400 & 20.24310 \\
4 & 0.89344 & 0.89624 & 22 & 21.46700 & 21.81300 \\
5 & 1.35725 & 1.39404 & 23 & 22.94280 & 23.39600 \\
6 & 2.12951 & 1.99635 & 24 & 24.44030 & 24.98820 \\
7 & 2.98573 & 2.69962 & 25 & 25.95870 & 26.58590 \\
8 & 3.91334 & 3.49978 & 26 & 27.49700 & 28.18600 \\
9 & 4.40970 & 4.39225 & 27 & 29.05460 & 29.78580 \\
10 & 5.94841 & 5.37202 & 28 & 30.63070 & 31.38330 \\
11 & 7.04344 & 6.43371 & 29 & 32.22460 & 32.97700 \\
12 & 8.18382 & 7.57163 & 30 & 33.83580 & 34.56580 \\
13 & 9.36580 & 8.77987 & 31 & 35.46370 & 36.14940 \\
14 & 10.58620 & 10.05230 & 32 & 37.10770 & 37.72780 \\
15 & 11.84230 & 11.38280 & 33 & 38.76730 & 39.3014 \\
16 & 12.81150 & 12.76510 & 34 & 40.44210 & 40.87120 \\
17 & 14.45250 & 14.19300 & 35 & 42.13150 & 42.43870 \\
18 & 15.80260 & 15.66050 & 36 & 43.83530 & 44.00550 \\
\hline
\end{tabular}




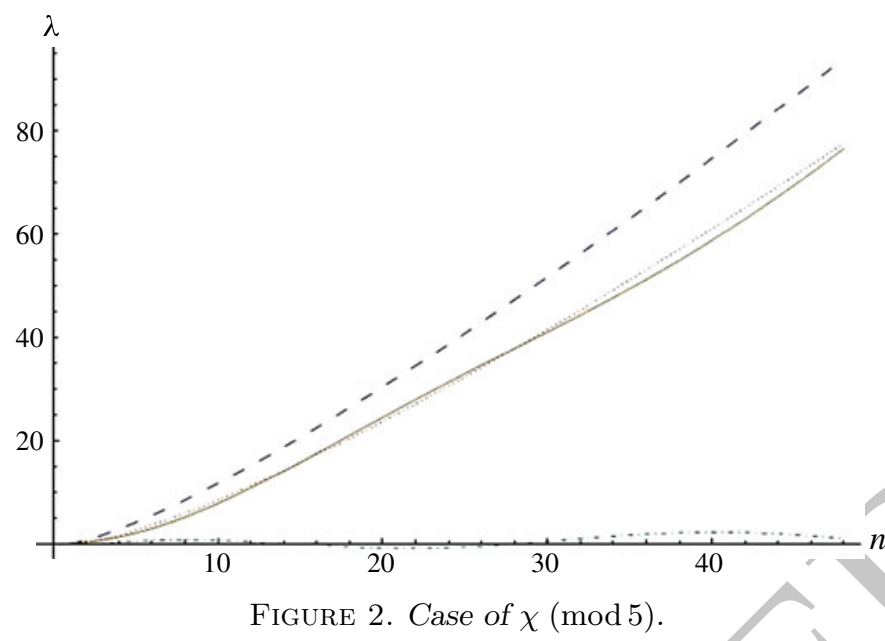

\begin{tabular}{|c|c|c|c|c|c|}
\hline \multicolumn{6}{|c|}{$\chi(\bmod 5)$} \\
\hline$n$ & $\tilde{\lambda}_{\chi}(n, M)$ & $\lambda_{\chi}(n, N)$ & & 1) & $\lambda_{\chi}(n, N)$ \\
\hline 1 & 0.13183 & 0.08562 & 21 & 25.37770 & 26.21450 \\
\hline 2 & 0.29872 & 0.34152 & 22 & 27.08610 & 27.92160 \\
\hline 3 & 0.91468 & 0.76482 & 23 & 28.81730 & 29.60960 \\
\hline 4 & 1.58476 & 1.35081 & 24 & 30.57020 & 31.27780 \\
\hline 5 & 2.63432 & 2.09300 & 25 & 32.34400 & 32.92720 \\
\hline 6 & 3.66199 & & 26 & 34.13770 & 34.56020 \\
\hline 7 & 4.77362 & 4.01225 & 27 & 35.95070 & 36.18030 \\
\hline 8 & 5.95664 & 5.16902 & 28 & 37.78220 & 37.79200 \\
\hline 9 & 7.06010 & 6.44188 & 29 & 39.63160 & 39.40090 \\
\hline 10 & 8.50254 & 7.81828 & 30 & 41.49820 & 41.01320 \\
\hline 11 & 9.85298 & 9.28519 & 31 & 43.38150 & 42.63540 \\
\hline 12 & 11.24880 & 10.82930 & 32 & 45.28090 & 44.2746 \\
\hline 13 & 12.68620 & 12.43740 & 33 & 47.19590 & 45.93760 \\
\hline 14 & 14.16200 & 14.09650 & 34 & 49.12610 & 47.63100 \\
\hline 15 & 15.67350 & 15.79410 & 35 & 51.07100 & 49.36130 \\
\hline 16 & 17.68370 & 17.51860 & 36 & 53.03020 & 51.13410 \\
\hline 17 & 14.45250 & 19.25930 & 37 & 55.00320 & 52.95430 \\
\hline 18 & 20.40000 & 21.00670 & 38 & 56.98980 & 54.82600 \\
\hline 19 & 22.03340 & 22.75260 & 39 & 58.98950 & 56.75210 \\
\hline 20 & 23.69300 & 24.49030 & 40 & 61.00210 & 58.73450 \\
\hline
\end{tabular}




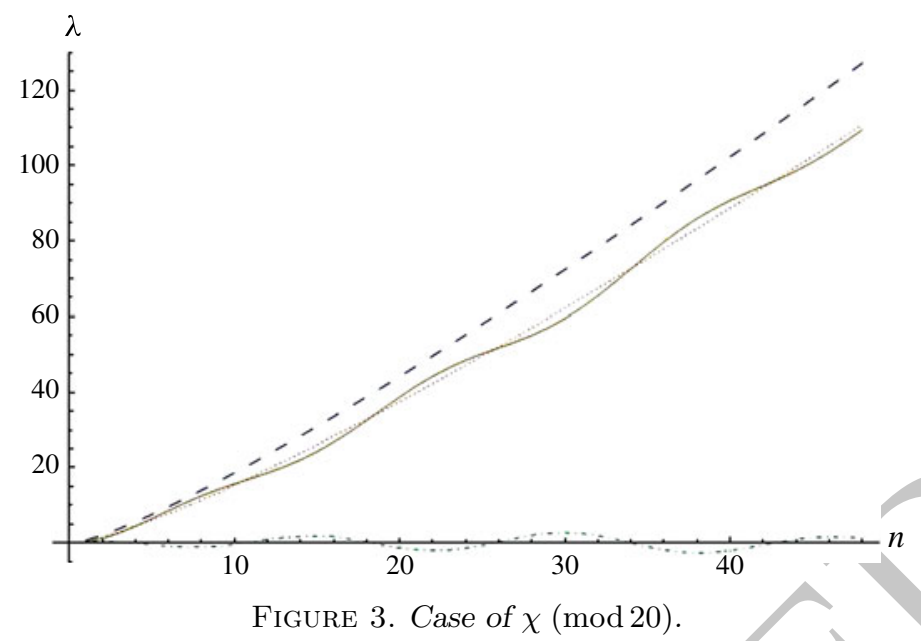

\begin{tabular}{rrrrrl}
\hline \multicolumn{5}{c}{$\chi(\bmod 20)$} & \\
\hline$n$ & $\tilde{\lambda}_{\chi}(n, M)$ & $\lambda_{\chi}(n, N)$ & $n$ & $\tilde{\lambda}_{\chi}(n, M)$ & $\lambda_{\chi}(n, N)$ \\
\hline 1 & 0.695021 & 0.319128 & 21 & 39.93370 & 41.70260 \\
2 & 1.68502 & 1.24419 & 22 & 42.33530 & 44.31350 \\
3 & 2.99412 & 2.68343 & 23 & 44.75970 & 46.59570 \\
4 & 4.48123 & 4.50032 & 24 & 47.20580 & 48.55720 \\
5 & 6.10005 & 6.53527 & 25 & 49.67270 & 50.26430 \\
6 & 7.82087 & 8.63067 & 26 & 52.15960 & 51.83150 \\
7 & 9.62565 & 10.65500 & 27 & 54.66570 & 53.403100 \\
8 & 11.50180 & 12.52230 & 28 & 57.19040 & 55.12930 \\
9 & 13.32220 & 14.20280 & 29 & 59.73290 & 57.14130 \\
10 & 15.43400 & 15.72450 & 30 & 62.29260 & 59.52940 \\
11 & 17.47760 & 17.16450 & 31 & 64.86910 & 62.32740 \\
12 & 19.56650 & 18.63130 & 32 & 67.46160 & 65.50710 \\
13 & 21.69710 & 20.24300 & 33 & 70.06980 & 68.98220 \\
14 & 23.86610 & 22.10320 & 34 & 72.69310 & 72.62260 \\
15 & 26.07070 & 24.28030 & 35 & 75.33110 & 76.27560 \\
16 & 28.54690 & 26.79300 & 36 & 77.98350 & 79.79060 \\
17 & 30.57800 & 29.60520 & 37 & 80.64970 & 83.04340 \\
18 & 32.87670 & 32.63050 & 38 & 83.32940 & 85.95580 \\
19 & 35.20320 & 35.74610 & 39 & 86.02230 & 88.50750 \\
20 & 37.55600 & 38.81360 & 40 & 88.72800 & 90.73760 \\
\hline & & & & &
\end{tabular}




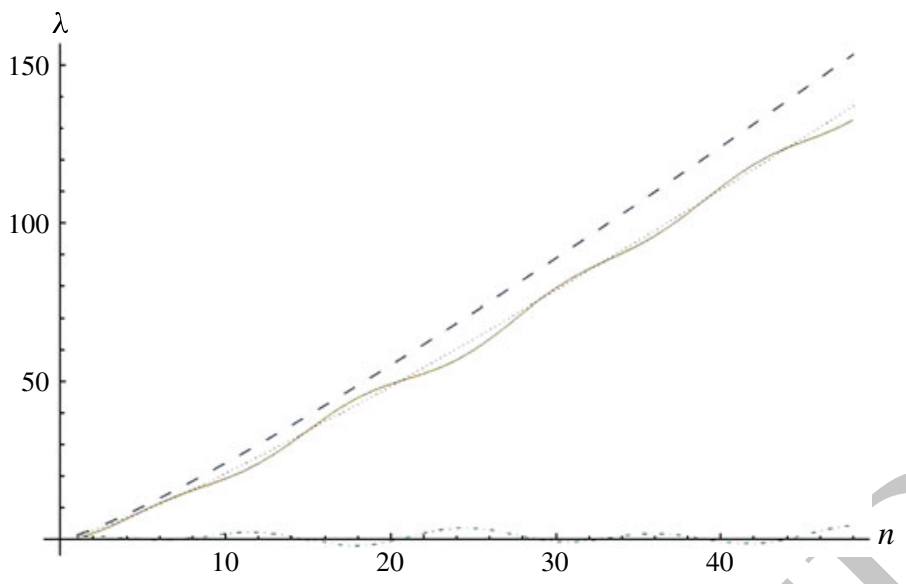

Figure 4. Case of $\chi(\bmod 60)$.

\begin{tabular}{rrrrrr}
\hline \multicolumn{5}{c}{$\chi(\bmod 60)$} & \\
\hline$n$ & $\tilde{\lambda}_{\chi}(n, M)$ & $\lambda_{\chi}(n, N)$ & $n$ & $\tilde{\lambda}_{\chi}(n, M)$ & $\lambda_{\chi}(n, N)$ \\
\hline 1 & 1.12226 & 0.48626 & 21 & 51.46920 & 50.88960 \\
2 & 2.78363 & 1.86950 & 22 & 54.42010 & 52.52830 \\
3 & 4.64204 & 3.94169 & 23 & 57.39370 & 54.44350 \\
4 & 6.83662 & 6.41363 & 24 & 60.38910 & 56.86290 \\
5 & 8.84658 & 8.98530 & 25 & 63.40530 & 59.89590 \\
6 & 11.11670 & 11.41720 & 26 & 66.44150 & 63.50750 \\
7 & 13.47080 & 13.58380 & 27 & 69.49700 & 67.53000 \\
8 & 15.89630 & 15.49640 & 28 & 72.57090 & 71.70750 \\
9 & 18.06830 & 17.28820 & 29 & 75.6628 & 75.7637 \\
10 & 20.92710 & 19.16770 & 30 & 78.77180 & 79.47310 \\
11 & 23.52000 & 21.35250 & 31 & 81.89750 & 82.71770 \\
12 & 26.15820 & 24.00100 & 32 & 85.03940 & 85.51520 \\
13 & 28.83810 & 27.16160 & 33 & 88.19690 & 88.00960 \\
14 & 31.55630 & 30.75170 & 34 & 91.36950 & 90.42920 \\
15 & 34.31030 & 34.57380 & 35 & 94.55690 & 93.02160 \\
16 & 37.56690 & 38.36300 & 36 & 97.75850 & 95.98430 \\
17 & 39.91620 & 41.85530 & 37 & 100.97400 & 99.40850 \\
18 & 42.76420 & 44.85610 & 38 & 104.20300 & 103.25300 \\
19 & 45.64000 & 47.29300 & 39 & 107.44500 & 107.35200 \\
20 & 48.54210 & 49.23760 & 40 & 110.70000 & 111.46000 \\
\hline & & & & & \\
\hline
\end{tabular}




\section{References}

1. S. Omar, R. Ouni and K. Mazhouda, 'On the zeros of Dirichlet L-functions', LMS J. Comput. Math. 14 (2011) 140-154; https://doi.org/10.1112/s1461157010000215.

2. F. Brown, 'Li's criterion and zero-free regions of $L$-functions', J. Number Theory 111 (2005) 1-32.

3. M. Coffey, 'Toward verification of the Riemann hypothesis: application of the Li criterion', Math. Phys. Anal. Geom. 8 (2005) no. 3, 211-255.

4. H. Davenport, Multiplicative number theory (Springer, New York, 1980).

5. X. Gourdon, 'The $10^{13}$ first zeros of the Riemann zeta function, and zeros computation at very large height', available at http://numbers.computation.free.fr/Constants/Miscellaneous/zetazeros1e13-1e24.pdf, October 2004.

6. W. Koepf and D. Schmersau, 'Bounded nonvanishing functions and Bateman functions', Complex Var. 25 (1994) 237-259.

7. X.-J. LI, 'The positivity of a sequence of numbers and the Riemann hypothesis', J. Number Theory 65 (1997) no. 2, 325-333

8. X.-J. LI, 'Explicit formulas for Dirichlet and Hecke L-functions', Illinois J. Math. 48 (2004) no. 2, $491-503$.

9. K. MAŚLANKA, 'Li's criterion for the Riemann hypothesis — numerical approach', Opuscula Math. 24 (2004) no. 1, 103-114.

10. S. Omar and S. Bounanan, 'Li's criterion and the Riemann hypothesis for function fields', Finite Fields Appl. 16 (2010) no. 6, 477-485.

11. S. Omar and K. Mazhouda, 'Le critère de Li et l'hypothèse de Riemann pour la classe de Selberg', J. Number Theory 125 (2007) no. 1, 50-58.

12. S. OMAR and K. MAZHoudA, "Corrigendum et addendum à "Le critère de Li et l'hypothèse de Riemann pour la classe de Selberg" [J. Number Theory 125 (2007) no. 1, 50-58]', J. Number Theory 130 (2010) no. 4, 1109-1114.

13. S. Omar and K. Mazhouda, 'The Li criterion and the Riemann hypothesis for the Selberg class II', J. Number Theory 130 (2010) no. 4, 1098-1108.

14. S. Omar, R. Ouni and K. Mazhouda, 'On the zeros of Hecke L-functions', Preprint, 2011.

\section{Sami Omar}

Department of Mathematics

King Khalid University

Abha 9004

Saudi Arabia

and

Department of Mathematics

Faculty of Science of Tunis

2092 Tunis

Tunisia

sami.omar@fst.rnu.tn

Kamel Mazhouda

Department of Mathematics

Faculty of Science of Monastir

5000 Monastir

Tunisia

kamel.mazhouda@fsm.rnu.tn
Raouf Ouni

Department of Mathematics

Faculty of Science of Tunis

2092 Tunis

Tunisia

raouf.ouni@fst.rnu.tn 